\title{
COORDINATED INVENTORY PLANNING FOR NEW AND OLD PRODUCTS UNDER WARRANTY
}

\author{
Wei Huang and Vidhyadhar Kulkarni \\ Department of Statistics and Operations Research \\ University of North Carolina \\ Chapel Hill, NC 27599 \\ JAYASHANKaR M. SWAMINATHAN \\ Department of Statistics and Operations Research \\ University of North Carolina \\ Chapel Hill, NC 27599 \\ and \\ The Kenan-Flagler Business School \\ University of North Carolina \\ Chapel Hill, NC 27599 \\ E-mail: Wei.Huang@sas.com; vkulkarn@email.unc.edu; msj@unc.edu
}

\begin{abstract}
In this article we study a firm that is facing demand from two sources: demand for new items and demand to replace failed items under warranty. We model this setting as a multiperiod single-product inventory problem where the demands for new items in different periods are independent and the demands for replacing failed items depend on the number of the items under warranty. We consider backlogging and emergency supply cases and study both discounted-cost and average-cost criteria. We prove the optimality of the $w$-dependent base stock ordering policy, where the base stock level is a function of $w$, the number of items currently under warranty. For the special case where the demand for new products is stationary, we prove the optimality of a stationary $w$-dependent base stock policy for the finitehorizon discounted-cost and the infinite-horizon discounted- and average-cost cases. We compare the integrated inventory policy with the one that neglects demands from items under warranty.
\end{abstract}

\section{INTRODUCTION}

After-sale parts and services are becoming an important aspect of closed-loop supply chains (see Guide and Wassenhove [7]). In cases where the original equipment 
manufacturer (OEM) manages the inventory for the product under warranty, the manufacturer needs to synergize inventory planning activities across new demand and demand arising from products under warranty.

In this article we consider a discrete-time multiperiod inventory model that jointly manages the inventory requirements for new products and warranty claims. Throughout this article we will restrict our attention to a single product. We assume that the demand in each period for the new product is stochastic and independent (not necessarily identical), whereas warranty claims are proportional to the number of products currently under warranty. Further, a fixed fraction of products under warranty in the field go out of warranty every period. The ordering, holding, and penalty costs are assumed to be linear and there is no delivery lag. We study the standard backlogging case and also the emergency supply case (with no backlogging) where the demand that cannot be satisfied by items in stock has to be satisfied by ordering from an emergency supplier. We prove that there exist functions $S_{n}(w)$ so that it is optimal to order up to $S_{n}(w)$ in period $n$ if there are $w$ items under warranty at that time. We call this a $w$-dependent base stock policy. The critical value $S_{n}(w)$ can be explicitly obtained by solving for the root of a single transcendental equation. For the special case where the demand for new products is stationary, we prove the optimality of a stationary $w$-dependent base stock policy for the finite-horizon discounted-cost and the infinite-horizon discounted- and averagecost cases. In our computational study, we compare the performance of such an integrated policy to a policy that only takes new demand into consideration while planning inventory. Our study indicates that, on average, 53\% cost improvements can be obtained from using the optimal integrated policy.

The rest of the article is organized as follows. We include a brief literature review of related research in Section 2. We formulate the problem in backlogging case in Section 3. We study the structure of the optimal inventory policy for the finite-horizon problem in Section 4, infinite-horizon discounted problem in Section 5, and long-run average-cost problem in Section 6. In Section 7 we study the extensions of the failure models and warranty expiration models. In Section 8 we formulate the problem in the emergency supply case and study the optimal inventory policy for the finite-horizon problem. In Section 9 we provide computational insights. We provide extensions and conclusions in Section 10.

\section{LITERATURE REVIEW}

There are three streams of research that are related to our inventory-warranty model. The first stream of research focuses on the effects of warranty under deterministic demand conditions. Porteus [12] considered a lot-sizing problem where the process goes out of control with a given probability each time it produces an item. He showed that the optimal lot size is smaller than the classical economic manufacturing quantity. Djamaludin, Murthy, and Wilson [5] and Wang and Sheu [15] studied other extensions of this scenario to find the optimal lot size taking into account long-run production inventory and warranty costs. As opposed to this stream, our focus is on periodic review warranty systems with stochastic demands. 
The second stream of literature has studied production systems with inventorydependent deterministic demand without warranty considerations. Khmelnitsky and Gerchak [10] studied a continuous review deterministic inventory model where demand rates can vary over time, shortages are possible, and the system has finite production capacity and find the optimal production control polices for such a system. Baker and Urban [1] analyzed the continuous, deterministic case of an inventory system in which the demand rate of an item is of a polynomial functional form, dependent on the inventory level. They developed the optimal policy to maximize average profit per unit time. In our work, the future requirement of a product is not only dependent on current inventory but also on previous sales.

The third stream of research considers inventory planning in a periodic setting under stochastic demand (see Swaminathan and Tayur [14] for a recent review). Within that stream of articles, those that consider return or remanufacturing are related to our work. Cohen, Nahmias, and Pierskalla [3] assumed that a fixed fraction of the products issued in a given period is returned after a fixed sojourn time in the market and might subsequently be reused. Optimality of a periodic review orderup-to policy is claimed assuming zero fixed cost and zero procurement lead times. Kelle and Silver [9] extended this approach by allowing for fixed order costs and stochastic sojourn time in the market. They proposed an approximation scheme transforming this model into a classical dynamic lot-sizing problem. Yuan and Cheung [16] proposed for this model an $(s, S)$-reorder policy based on the sum of the on-hand stock and the number of items in the market. The single-stage remanufacturing system was first studied by Simpson [13] and Inderfurth [8]. Simpson [13] established the optimality of a three-parameter policy consisting of remanufactureup-to, order-up-to, and dispose-down-to levels. Inderfurth [8] extended those results to the case of positive but identical lead times for ordering and remanufacturing and argued that if lead times are not identical, then the optimal policy will be more complicated. More recently, Feinberg and Lewis [6] considered a single commodity inventory system in which the demand is modeled by a sequence of independent and identically distributed (i.i.d.) random variables that can take negative values (thereby modeling some of the remanufacturing or product return settings). A multiechelon remanufacturing system has been studied by DeCroix [4]. As opposed to this stream of research where remanufacturing or returns increase supply, in our model the demand increases when there are warranty claims. This creates additional dependence between sales in the past and demand in the future, making the analysis complicated.

\section{MODEL WITH BACKLOGGING}

In our model, inventory for a single product is managed for multiple periods. The firm offers replacement of items that fail under warranty. The demand arises from two sources: new demand and demand to replace failed items under warranty. Let $\zeta_{n}$ be the new demand in period $n$. Let $F_{n}(\cdot)$ be its cumulative distribution function (c.d.f.), and $f_{n}(\cdot)$ be its probability density function (p.d.f.). Let $X_{n}$ be the inventory 
on hand and let $W_{n}$ be the number of items under warranty in period $n$. In period $n$, we decide to order an amount $A_{n}$ and define $Y_{n}=X_{n}+A_{n}$. We treat $Y_{n} \geq X_{n}$ as the decision variable in period $n$. The delivery is assumed to be instantaneous, so that $Y_{n}$ is the amount available to satisfy the demand for new and warranty claims in period $n$. Any demand that cannot be immediately satisfied is backlogged.

We assume that the warranty is renewable (i.e., the warranty period of the replaced item starts afresh). Such warranty models have been studied in the past (see Blischke and Murthy [2]). Also, we assume that the demand to replace failed items is based on a fixed proportional model (i.e., a fixed fraction $\beta$ of the items under warranty fail in any given period). Furthermore, in period $n$, a fixed fraction $\delta$ of the items under warranty remains in warranty and the remaining fraction $(1-\delta)$ of the items goes out of warranty. These are only approximations to the true system in which the number of failures and the number of items remaining under warranty depend exclusively on the warranty time and age distribution of items in the field. These assumptions keep the analysis tractable. We will treat the model with random $\beta$ and $\delta$ in Section 7. Under these assumptions, we get

$$
\begin{aligned}
& W_{n+1}=\delta\left[(1-\beta) W_{n}+\min \left(Y_{n}, \beta W_{n}+\zeta_{n}\right)+\left[X_{n}\right]^{-}\right], \\
& X_{n+1}=Y_{n}-\zeta_{n}-\beta W_{n},
\end{aligned}
$$

where $\left[X_{n}\right]^{-}=-\min \left(X_{n}, 0\right)$. Thus, $\left\{\left(\left(W_{n}, X_{n}\right), Y_{n}\right), n \geq 0\right\}$ is a Markov decision process. The term $\left[X_{n}\right]^{-}$in (1) accounts for the fact that when backorders are filled, they get added to the items under warranty.

Next we describe the cost structure. We assume that there is a per-unit procurement cost $c$, holding cost $h$ for each item remaining at the end of a period, and shortage cost $p$ for each unit of backlogged demand in any period. Let

$L_{n}(w, y)=p \int_{\zeta=y-\beta w}^{\infty}(\zeta-y+\beta w) f_{n}(\zeta) d \zeta+h \int_{\zeta=0}^{y-\beta w}(y-\zeta-\beta w) f_{n}(\zeta) d \zeta$

represent the expected penalty and holding cost incurred in period $n$ if $W_{n}=w$ and $Y_{n}=y$. The total one-period expected cost incurred for ordering up to $y \geq x$ is given by

$$
C_{n}(w, x, y)=c(y-x)+L_{n}(w, y) .
$$

Let $\alpha, 0 \leq \alpha \leq 1$, be the discount factor. Let $\pi$ be any policy for choosing decision $Y_{n}$ at time $n$, based on the history up to time $n$. We consider three objective functions for choosing an optimal policy as described below.

\subsection{Finite Horizon}

Under this objective function we try to minimize the expected total discounted cost (ETDC) over periods $0,1,2, \ldots, N$. Let $V_{N}^{\pi}(w, x)$ be the ETDC of following the policy $\pi$ over periods $0,1, \ldots, N$. Since we have a finite horizon, we need to specify 
the terminal cost. Let $T(w, x)$ be the terminal cost at time $N$ if $W_{N}=w$ and $X_{N}=x$. Thus,

$$
V_{N}^{\pi}(w, x)=E_{\pi}\left(\sum_{n=0}^{N-1} \alpha^{n} C_{n}\left(W_{n}, X_{n}, Y_{n}\right)+\alpha^{N} T\left(W_{N}, X_{N}\right) \mid W_{0}=w, X_{0}=x\right) .
$$

Here $E_{\pi}$ denotes the expectation under the assumption that the policy $\pi$ is followed. Let $V_{N}(w, x)$ be the optimal ETDC of operating the system over period $0, \ldots, N$; that is,

$$
V_{N}(w, x)=\inf _{\pi} V_{N}^{\pi}(w, x)
$$

A policy $\pi^{*}$ is called optimal for finite-horizon ETDC if

$$
V_{N}(w, x)=V_{N}^{\pi^{*}}(w, x) \text { for all } w \text { and } x .
$$

\subsection{Infinite-Horizon ETDC}

The second objective function is to minimize the ETDC over the infinite horizon. In this case, there is no terminal cost function. Let $V^{\pi}(w, x)$ be the infinite-horizon ETDC of following policy $\pi$; that is,

$$
V^{\pi}(w, x)=E_{\pi}\left(\sum_{n=0}^{\infty} \alpha^{n} C_{n}\left(W_{n}, X_{n}, Y_{n}\right) \mid W_{0}=w, X_{0}=x\right) .
$$

Similarly, let $V(w, x)$ be the optimal infinite-horizon ETDC; that is,

$$
V(w, x)=\inf _{\pi} V^{\pi}(w, x) .
$$

A policy $\pi^{*}$ is called optimal for infinite-horizon ETDC if

$$
V(w, x)=V^{\pi^{*}}(w, x) \text { for all } w \text { and } x .
$$

\subsection{Infinite-Horizon Average Cost}

Let $g^{\pi}(w, x)$ be the expected cost per period of following policy $\pi$ over the infinite horizon starting from state $(w, x)$. Again, there is no terminal cost in this formulation. Thus, assuming that the limit exists,

$$
g^{\pi}(w, x)=\lim _{N \rightarrow \infty} \frac{1}{N+1} E_{\pi}\left(\sum_{n=0}^{N} C_{n}\left(W_{n}, X_{n}, Y_{n}\right) \mid W_{0}=w, X_{0}=x\right) .
$$

Typically, this limit is independent of the starting state $(w, x)$. Let $g(w, x)$ be the optimal expected cost per period over the infinite horizon starting in state $(w, x)$; that is, 


$$
g(w, x)=\inf _{\pi} g^{\pi}(w, x) .
$$

Typically this infimum does not depend on the initial state $(w, x)$. A policy $\pi^{*}$ is called optimal for infinite-horizon average cost if

$$
g(w, x)=g^{\pi^{*}}(w, x) \text { for all } w \text { and } x .
$$

We obtain optimal policies under all three objective functions, beginning with the finite-horizon ETDC in the next section.

\section{FINITE HORIZON}

In this section we study the finite-horizon problem with $N$ periods and terminal cost $T(w, x)$ and show how to compute $V_{N}(w, x)$ of $(5)$. First, define $V_{n, N}(w, x)$ to be the optimal ETDC over periods $n, n+1, \ldots, N$ starting with $W_{n}=w$ and $X_{n}=x$. Let $G_{n, N}(w, x, y)$ be the ETDC over periods $n, n+1, \ldots, N$ given $W_{n}=w, X_{n}=x$, and $Y_{n}=y$. Then the standard dynamic programming (DP) recursion yields

$$
\begin{aligned}
V_{N, N}(w, x)= & T(w, x), \\
G_{n, N}(w, x, y)= & c y-c x+\alpha L_{n}(w, y) \\
& +\alpha \int_{0}^{y-\beta w} V_{n+1, N}\left(\delta\left(w+\zeta+[x]^{-}\right), y-\beta w-\zeta\right) f_{n}(\zeta) d \zeta \\
& +\alpha \int_{y-\beta w}^{\infty} V_{n+1, N}\left(\delta\left((1-\beta) w+y+[x]^{-}\right),\right. \\
y-\beta w-\zeta) f_{n}(\zeta) d \zeta, & n=0,1, \ldots, N-1, \\
V_{n, N}(w, x)= & \min _{y \geq x}\left\{G_{n, N}(w, x, y)\right\}, \quad n=0,1, \ldots, N-1,
\end{aligned}
$$

where $L_{n}(w, y)$ is as in (2). Then we have

$$
V_{N}(w, x)=V_{0, N}(w, x) .
$$

We will show that with a judicious choice of the terminal cost function $T$, the value of $y$ that minimizes the function $G_{n, N}(w, y, x)$ is in fact independent of $x$. One such special terminal cost function is given by

$$
T(w, x)=-c x+\frac{c \beta \alpha \delta[x]^{-}}{1-\alpha \delta}+\frac{c \beta w}{1-\alpha \delta}
$$

The above terminal cost is easily explained if we assume that any leftover inventory at period $N$ can be returned at the original purchase price $c$ and any backlog has 
to be satisfied at purchase $\operatorname{cost} c$. Furthermore, all of the warranty claims arising out of the $w$ items under warranty and the $[x]^{-}$items sold at time $N$ have to be covered. With this terminal operation, the first term in (15) reflects the cost of processing the terminal inventory $x$. The second term represents the expected discounted cost of warranty claims of $[x]^{-}$items sold at period $N$, since these products will be introduced into the field only in the next period. The third term represents the similar warranty cost for the $w$ items under warranty at period $N$.

Now let

$$
\bar{p}=p-\frac{(1-\alpha) c \delta \beta}{1-\alpha \delta}
$$

For $n=0,1, \ldots, N-1$, define

$$
\begin{aligned}
S_{n}(w)= & \beta w+F_{n}^{-1}\left(\frac{\bar{p}-c(1-\alpha)}{\bar{p}+h}\right) \\
\bar{L}_{n}(w, y)= & h \int_{0}^{y-\beta w}(y-\beta w-\zeta) f_{n}(\zeta) d \zeta+\bar{p} \int_{y-\beta w}^{\infty}(\zeta-y+\beta w) f_{n}(\zeta) d \zeta, \\
\tau_{n}(w, y)= & y-\beta w-\beta \delta w-F_{n}^{-1}\left(\frac{\bar{p}-c(1-\alpha)}{\bar{p}+h}\right) \\
\Delta_{n}= & \alpha\left(\frac{c \beta}{1-\alpha \delta} \delta+c\right) \mu_{n}+c(1-\alpha) F_{n}^{-1}\left(\frac{\bar{p}-c(1-\alpha)}{\bar{p}+h}\right) \\
& +\alpha \bar{L}_{n}\left(w, S_{n}(w)\right) \\
\mu_{n}= & E\left[\zeta_{n}\right]=\int_{0}^{\infty}\left(1-F_{n}(\zeta)\right) d \zeta .
\end{aligned}
$$

Here $\bar{L}_{n}$ is the same as the function $L_{n}$ of (2), with $\bar{p}$ replacing $p$. Note that $\Delta_{n}$ is in fact independent of $w$. This observation is crucial in deriving our results. Also, define

$$
\begin{aligned}
& H_{N}(w, x)=0, \\
& H_{n}(w, x)= \begin{cases}0 & \text { if } x \leq S_{n}(w) \\
G_{n, N}(w, x, x)-G_{n, N}\left(w, x, S_{n}(w)\right) & \text { otherwise }\end{cases} \\
& \text { for } 0 \leq n \leq N-1 .
\end{aligned}
$$


THEOREM 1: Suppose the demands in each period are stochastically increasing; that is,

$$
F_{n}(x) \geq F_{n+1}(x) \text { for } n=0,1, \ldots, N-2 \text {. }
$$

Then

(i)

$$
\begin{aligned}
G_{n, N}(w, x, y)= & c(1-\alpha) y-c x+\alpha \bar{L}_{n}(w, y) \\
& +\sum_{i=n+1}^{N-1} \alpha^{i-n} \Delta_{i}+\alpha\left(\frac{c \beta}{1-\alpha \delta} \delta+c \beta\right) w \\
& +\alpha\left(\frac{c \beta}{1-\alpha \delta} \delta+c\right) \mu_{n}+\alpha\left(\frac{c \beta}{1-\alpha \delta} \delta\right)[x]^{-} \\
& +\alpha \int_{0}^{\tau_{n+1}(w, y)} H_{n+1}\left(\delta\left(w+\min (y-\beta w, \zeta)+[x]^{-}\right),\right. \\
& y-\beta w-\zeta) f_{n}(\zeta) d \zeta
\end{aligned}
$$

for $n=0, \ldots, N-1$. Furthermore, $y=S_{n}(w)$ minimizes $G_{n}(w, x, y)$ for $n=0, \ldots, N-1$ and $G_{n, N}(w, x, y)$ increases with respect to $y$ when $y \geq S_{n}(w)$.

(ii)

$$
\int_{0}^{\tau_{n}(w, y)} H_{n}\left(\delta\left(w+\min (y-\beta w, \zeta)+[x]^{-}\right), y-\beta w-\zeta\right) f_{n-1}(\zeta) d \zeta
$$

is an increasing function of $y$ when $y \geq S_{n-1}(w)$ for a given $w$ for $n=$ $1, \ldots, N-1$.

(iii)

$$
V_{n, N}(w, x)=T(w, x)+\sum_{i=n}^{N-1} \alpha^{i-n} \Delta_{i}+H_{n}(w, x) \quad \text { for } n=0, \ldots, N-1
$$

The proof follows from a series of claims using backward induction.

Claim 1: Parts (i), (ii), and (iii) hold for $N-1$. 


\section{ProOF:}

$$
\begin{aligned}
G_{N-1, N}(w, x, y) & \\
= & c y-c x+\alpha L_{N-1}(w, y) \\
& +\alpha \int_{0}^{y-\beta w}\left[\frac{c \beta}{1-\alpha \delta} \delta\left(w+\zeta+[x]^{-}\right)-c(y-\beta w-\zeta)\right] f_{N-1}(\zeta) d \zeta \\
& +\alpha \int_{y-\beta w}^{\infty}\left[\frac{c \beta}{1-\alpha \delta} \delta\left(w+y-\beta w+[x]^{-}\right)\right. \\
& \left.\quad+\frac{c \beta \alpha}{1-\alpha \delta} \delta(\beta w+\zeta-y)-c(y-\beta w-\zeta)\right] f_{N-1}(\zeta) d \zeta \\
= & c(1-\alpha) y-c x+\alpha\left(\frac{c \beta}{1-\alpha \delta} \delta+c \beta\right) w+\alpha\left(\frac{c \beta}{1-\alpha \delta} \delta\right)[x]^{-} \\
& +\alpha\left(\frac{c \beta}{1-\alpha \delta} \delta+c\right) \mu_{N-1}+\alpha \bar{L}_{N-1}(w, y),
\end{aligned}
$$

where

$$
\begin{aligned}
\bar{L}_{N-1}(w, y)= & \left(p-\delta \frac{(1-\alpha) c \beta}{1-\alpha \delta}\right) \int_{y-\beta w}^{\infty}(\zeta-y+\beta w) f_{N-1}(\zeta) d \zeta \\
& +h \int_{0}^{y-\beta w}(y-\beta w-\zeta) f_{N-1}(\zeta) d \zeta \\
= & \bar{p} \int_{y-\beta w}^{\infty}(\zeta-y+\beta w) f_{N-1}(\zeta) d \zeta \\
& +h \int_{0}^{y-\beta w}(y-\beta w-\zeta) f_{N-1}(\zeta) d \zeta .
\end{aligned}
$$

It is easy to see that $y=S_{N-1}(w)=\beta w+F_{N-1}^{-1}((\bar{p}-c(1-\alpha)) /(\bar{p}+h))$ minimizes $G_{N-1, N}(w, x, y)$ for all $x$. Hence, $G_{N-1, N}(w, x, y)$ increases with respect to $y$ when $y \geq S_{N-1}(w)$. Therefore, we get

$$
V_{N-1, N}(w, x)= \begin{cases}G_{N-1, N}\left(w, x, S_{N-1}(w)\right) & \text { if } x \leq S_{N-1}(w) \\ G_{N-1, N}(w, x, x) & \text { otherwise. }\end{cases}
$$

Using the notation $\Delta_{N-1}$ from (18), it is easy to show that

$$
G_{N-1, N}\left(w, x, S_{N-1}(w)\right)=T(w, x)+\Delta_{N-1} .
$$


Hence, we can rewrite (23) as

$$
\begin{aligned}
V_{N-1, N}(w, x) & \\
= & T(w, x)+\Delta_{N-1} \\
& + \begin{cases}0 & \text { if } x \leq S_{N-1}(w) \\
G_{N-1, N}(w, x, x)-G_{N-1, N}\left(w, x, S_{N-1}(w)\right) & \text { otherwise }\end{cases} \\
= & T(w, x)+\Delta_{N-1}+H_{N-1}(w, x),
\end{aligned}
$$

where $H_{N-1}(w, x)$ is as given as (19).

Next we show that $\int_{0}^{\tau_{N-1}(w, y)} H_{N-1}\left(\delta\left(w+\min (y-\beta w, \zeta)+[x]^{-}\right), y-\beta w-\zeta\right)$ $f_{N-2}(\zeta) d \zeta$ is an increasing function of $y$, for $y \geq S_{N-2}(w)$. Taking the first-order derivative with respect to $y$, we get

$$
\begin{gathered}
\frac{\partial}{\partial y} \int_{0}^{\tau_{N-1}(w, y)} H_{N-1}\left(\delta\left(w+\zeta+[x]^{-}\right), y-\beta w-\zeta\right) f_{N-2}(\zeta) d \zeta \\
=\frac{\partial}{\partial y} \int_{0}^{\tau_{N-1}(w, y)}\left[G_{N-1, N}\left(\delta\left(w+\zeta+[x]^{-}\right), y-\beta w-\zeta, y-\beta w-\zeta\right)\right. \\
-G_{N-1, N}\left(\delta\left(w+\zeta+[x]^{-}\right),\right. \\
\left.\left.y-\beta w-\zeta, S_{N-1}\left(\delta\left(w+\zeta+[x]^{-}\right)\right)\right)\right] f_{N-2}(\zeta) d \zeta \\
=\int_{0}^{\tau_{N-1}(w, y)} G_{N-1, N}^{3}\left(\delta\left(w+\zeta+[x]^{-}\right), y-\beta w-\zeta, y-\beta w-\zeta\right) f_{N-2}(\zeta) d \zeta,
\end{gathered}
$$

where $G^{3}$ represents the first partial with respect to the third argument of $G$. Since $S_{N-1}(w)$ minimizes $G_{N-1, N}(w, x, y)$ and the definition of $H_{N-1}(w, x)$, we get

$$
\begin{gathered}
\left\{y: G_{N-1, N}^{3}\left(\delta\left(w+\zeta+[x]^{-}\right), y-\beta w-\zeta, y-\beta w-\zeta\right)>0\right\} \\
=\left\{y: H_{N-1}\left(\delta\left(w+\zeta+[x]^{-}\right), y-\beta w-\zeta\right)>0\right\} .
\end{gathered}
$$

From the assumption in (20) we get $S_{N-2}(w) \leq S_{N-1}(w)+\beta \delta w$. This proves that the quantity in (25) is nonnegative when $y \geq S_{N-2}(w)$.

Claim 2: If parts (i), (ii), and (iii) of Theorem 1 hold for $n \leq N-1$, then they hold for $n-1$. 
Proof: The induction hypothesis implies that we have $G_{n, N}(w, x, y)$

$$
\begin{aligned}
= & c(1-\alpha) y-c x+\alpha \bar{L}_{n}(w, y)+\sum_{i=n+1}^{N-1} \alpha^{i-n} \Delta_{i} \\
& +\alpha\left(\frac{c \beta}{1-\alpha \delta} \delta+c \beta\right) w+\alpha \frac{c \beta}{1-\alpha \delta} \delta[x]^{-}+\alpha\left(\frac{c \beta}{1-\alpha \delta} \delta+c\right) \mu_{n} \\
& +\alpha \int_{0}^{\infty} H_{n+1}\left(\delta\left(w+\min (y-\beta w, \zeta)+[x]^{-}\right), y-\beta w-\zeta\right) f_{n}(\zeta) d \zeta,
\end{aligned}
$$

$y=S_{n}(w)$ minimizes $G_{n, N}(w, x, y)$, and $G_{n, N}(w, x, y)$ increases with respect to $y$ when $y \geq S_{n}(w)$. Also, $\int_{0}^{\infty} H_{n}\left(\delta\left(w+\min (y-\beta w, \zeta)+[x]^{-}\right), y-\beta w-\zeta\right)$ $f_{n-1}(\zeta) d \zeta$ is an increasing function of $y$ when $y \geq S_{n-1}(w)$. We have

$$
\begin{aligned}
G_{n, N}\left(w, x, S_{n}(w)\right)= & \sum_{i=n+1}^{N-1} \alpha^{i-n} \Delta_{i}+\alpha\left(\frac{c \beta}{1-\alpha \delta} \delta+c \beta\right) w+\alpha\left(\frac{c \beta}{1-\alpha \delta} \delta+c\right) \mu_{n} \\
& +c(1-\alpha) S_{n}(w)+\alpha \bar{L}_{n}\left(w, S_{n}(w)\right)+\alpha \frac{c \beta}{1-\alpha \delta} \delta[x]^{-} \\
= & \frac{c \beta}{1-\alpha \delta} w+\alpha \frac{c \beta}{1-\alpha \delta} \delta[x]^{-}+\sum_{i=n}^{N-1} \alpha^{i-n} \Delta_{i}
\end{aligned}
$$

and, correspondingly,

$$
\begin{aligned}
V_{n, N}(w, x)= & T(w, x)+\sum_{i=n}^{N-1} \alpha^{i-n} \Delta_{i} \\
& + \begin{cases}0 & \text { if } x \leq S_{n}(w) \\
G_{n, N}(w, x, x)-G_{n, N}\left(w, x, S_{n}(w)\right) & \text { otherwise. }\end{cases}
\end{aligned}
$$

Therefore, from the DP recursion in (13) we get

$$
\begin{aligned}
G_{n-1, N}(w, x, y) \\
=c y-c x+\alpha L_{n-1}(w, y) \\
\quad+\alpha \int_{0}^{y-\beta w} V_{n, N}\left(\delta\left(w+\zeta+[x]^{-}\right), y-\beta w-\zeta\right) f_{n-1}(\zeta) d \zeta \\
\quad+\alpha \int_{y-\beta w}^{\infty} V_{n, N}\left(\delta\left(w+y-\beta w+[x]^{-}\right), y-\beta w-\zeta\right) f_{n-1}(\zeta) d \zeta \\
=c(1-\alpha) y-c x+\alpha \bar{L}_{n-1}(w, y)+\sum_{i=n}^{N-1} \alpha^{i-n+1} \Delta_{i}+\alpha\left(\frac{c \beta}{1-\alpha \delta} \delta+c \beta\right) w \\
\quad+\alpha\left(\frac{c \beta}{1-\alpha \delta} \delta+c\right) \mu_{n-1}+\alpha \frac{c \beta}{1-\alpha \delta} \delta[x]^{-} \\
\quad+\alpha \int_{0}^{\infty} H_{n}\left(\delta\left(w+\min (y-\beta w, \zeta)+[x]^{-}\right), y-\beta w-\zeta\right) f_{n-1}(\zeta) d \zeta,
\end{aligned}
$$


which implies that (i) holds for $n-1$. It is easy to see that $y=S_{n-1}(w)$ minimizes $c(1-\alpha) y+\alpha \bar{L}_{n-1}(w, y)$. Assumption (20) implies that

$$
F_{n-1}^{-1}\left(\frac{\bar{p}-c(1-\alpha)}{\bar{p}+h}\right) \leq F_{n}^{-1}\left(\frac{\bar{p}-c(1-\alpha)}{\bar{p}+h}\right),
$$

which shows that $\tau_{n}\left(w, S_{n-1}(w)\right)<0$. This proves that

$$
\int_{0}^{\tau_{n}\left(w, S_{n-1}(w)\right)} H_{n}\left(\delta\left(w+\zeta+[x]^{-}\right), S_{n-1}(w)-\beta w-\zeta\right) f_{n-1}(\zeta) d \zeta=0 .
$$

Then $y=S_{n-1}(w)$ minimizes $G_{n-1, N}(w, x, y)$. Hence, $G_{n-1, N}(w, x, y)$ increases with respect to $y$ when $y \geq S_{n-1}(w)$. Furthermore, the first-order derivative of $\int_{0}^{\infty} H_{n-1}\left(\delta\left(w+\min (y-\beta w, \zeta)+[x]^{-}\right), y-\beta w-\zeta\right) f_{n-2}(\zeta) d \zeta$ with respect to $y$ is given by

$$
\begin{aligned}
& \frac{\partial}{\partial y} \int_{0}^{\tau_{n-1}(w, y)} H_{n-1}\left(\delta\left(w+\zeta+[x]^{-}\right), y-\beta w-\zeta\right) f_{n-2}(\zeta) d \zeta \\
& =\frac{\partial}{\partial y} \int_{0}^{\tau_{n-1}(w, y)}\left[G_{n-1, N}\left(\delta\left(w+\zeta+[x]^{-}\right), y-\beta w-\zeta, y-\beta w-\zeta\right)\right. \\
& -G_{n-1, N}\left(\delta\left(w+\zeta+[x]^{-}\right),\right. \\
& \left.\left.y-\beta w-\zeta, S_{n-1}\left(\delta\left(w+\zeta+[x]^{-}\right)\right)\right)\right] f_{n-2}(\zeta) d \zeta \\
& =\int_{0}^{\tau_{n-1}(w, y)} G_{n-1, N}^{3}\left(\delta\left(w+\zeta+[x]^{-}\right), y-\beta w-\zeta, y-\beta w-\zeta\right) f_{n-2}(\zeta) d \zeta .
\end{aligned}
$$

Here the superscript 3 denotes the partial derivative of the function $g$ with respect to its third argument. Now the right-hand side is nonnegative when $y \geq S_{n-2}(w)$, since $G_{n-1, N}(w, x, y)$ increases with respect to $y$ when $y \geq S_{n-1}(w)$, and

$$
\begin{gathered}
\left\{y: G_{n-1, N}^{3}\left(\delta\left(w+\zeta+[x]^{-}\right), y-\beta w-\zeta, y-\beta w-\zeta\right)>0\right\} \\
=\left\{y: H_{n-1}\left(\delta\left(w+\zeta+[x]^{-}\right), y-\beta w-\zeta\right)>0\right\}
\end{gathered}
$$

and

$$
\tau_{n-1}\left(w, S_{n-2}(w)\right) \leq 0 .
$$

Thus, (ii) holds for $n-1$.

This implies that the base stock level policy, which orders up to $S_{n-1}(w)$, is the optimal for the discounted-cost function over $n-1, n, \ldots, N$ periods. Clearly, we get

$$
G_{n-1, N}\left(w, x, S_{n-1}(w)\right)=T(w, x)+\sum_{i=n-1}^{N-1} \alpha^{i-n+1} \Delta_{i}
$$


Therefore,

$$
\begin{aligned}
V_{n-1, N}(w, x)= & T(w, x)+\sum_{i=n-1}^{N-1} \alpha^{i-n+1} \Delta_{i} \\
& + \begin{cases}0 & \text { if } x \leq S_{n-1}(w) \\
G_{n-1, N}(w, x, x)-G_{n-1, N}\left(w, x, S_{n-1}(w)\right) & \text { otherwise }\end{cases}
\end{aligned}
$$

which implies that (iii) holds for $n-1$. Theorem 1 follows from this.

This yields the main result as stated in the following theorem. It is an immediate result of Theorem 1 .

THEOREM 2: Let $S_{n}(w)$ be as given in (17). It is optimal to order up to $S_{n}(w)$ in period $n$ if $W_{n}=w$ and $X_{n}=x$.

We call the policy in Theorem 2 a $w$-dependent base stock policy.

Special Case (i.i.d. Demands): Consider the special case where $F_{n}(x)=F(x)$ for $n=0,1, \ldots, N-1$; that is, the demands are i.i.d. In this case, the base stock level in period $n$ is given by

$$
S(w)=\beta w+F^{-1}\left(\frac{\bar{p}-c(1-\alpha)}{\bar{p}+h}\right)
$$

for all $n=0, \ldots, N-1$. Thus, the stationary $w$-dependent base stock policy is optimal in this case. It is unusual to get a stationary optimal policy for a finite-horizon problem. However, the special structure of our terminal cost facilitates this result.

\section{INFINITE-HORIZON DISCOUNTED COST}

In this section we study the infinite-horizon discounted-cost problem with i.i.d. demands. In Section 4 we have proved that in the i.i.d. demands case, the stationary $w$-dependent base stock policy minimizes the total expected discounted (or undiscounted) cost over any finite horizon $N$. We denote this stationary policy by $\pi^{*}$. Thus, we have shown that

$$
\begin{aligned}
V_{N}(w, x) & =V_{N}^{\pi^{*}}(w, x) \\
& =E_{\pi^{*}}\left(\sum_{n=1}^{N-1} \alpha^{n} C_{n}\left(W_{n}, X_{n}, Y_{n}\right)+\alpha^{N} T\left(W_{n}, X_{n}\right) \mid W_{0}=w, X_{n}=0\right) .
\end{aligned}
$$

In this section we will show that

$$
\lim _{N \rightarrow \infty} E_{\pi^{*}}\left(\alpha^{N} T\left(W_{N}, X_{N}\right) \mid W_{0}=w, X_{n}=0\right)=0 ;
$$


that is, $\pi^{*}$ also minimizes the expected total discounted cost over the infinite horizon. Our main result is given in the next theorem.

Theorem 3: Suppose $F_{n}(x)=F(x)$ for all $n \geq 0$. Then the stationary $w$-dependent base stock policy that orders up to $S(w)=\beta w+F^{-1}((\bar{p}-c(1-\alpha)) /(\bar{p}+h))$ in any period in state $(w, x)$ minimizes the infinite-horizon discounted cost.

Proof: From (1), we get

$$
W_{n+1} \leq \delta\left[(1-\beta) W_{n}+\beta W_{n}+\zeta_{n}+\left[X_{n}\right]^{-}\right] .
$$

Hence, taking expected values,

$$
\begin{aligned}
E_{\pi^{*}}\left(W_{n+1}\right) & \leq \delta E_{\pi^{*}}\left(W_{n}\right)+\delta \mu+\delta E_{\pi^{*}}\left(\left[X_{n}\right]^{-}\right), \\
& \leq \delta E_{\pi^{*}}\left(W_{n}\right)+2 \delta \mu
\end{aligned}
$$

where $\mu=E\left(\zeta_{n}\right)$. Iterating the above we get

$$
\begin{aligned}
E_{\pi^{*}}\left(W_{N}\right) & \leq \delta^{N} E_{\pi^{*}}\left(W_{0}\right)+2\left(\delta+\cdots+\delta^{N}\right) \mu \\
& \leq \delta^{N} w+\frac{2 \delta \mu\left(1-\delta^{N}\right)}{1-\delta} \\
& \leq \delta^{N} w+\frac{2 \mu}{1-\delta} .
\end{aligned}
$$

The effect of the initial inventory $X_{0}=x$ is to increase the above right-hand side at most by $\delta^{N} x$. Also, $E_{\pi^{*}}\left(X_{N}\right) \geq-\mu$. Combining the above arguments we get

$$
\begin{aligned}
E_{\pi^{*}}\left(T\left(W_{N}, X_{N}\right) \mid W_{0}=w, X_{0}=x\right) & =E_{\pi^{*}}\left(\frac{c \beta}{1-\delta} W_{N}-c X_{N} \mid W_{0}=w, X_{0}=x\right) \\
& \leq\left(\frac{2 \mu}{1-\delta}+(w+x) \delta^{N}\right) \frac{c \beta}{1-\delta}+c \mu .
\end{aligned}
$$

Hence,

$$
\lim _{N \rightarrow \infty} \alpha^{N} E_{\pi^{*}}\left(T\left(W_{N}, X_{N}\right) \mid W_{0}=w, X_{0}=x\right)=0
$$

Hence, it follows that

$$
\lim _{N \rightarrow \infty} V_{N}^{\pi^{*}}(w, x)=V^{\pi^{*}}(w, x)=V(w, x)
$$

that is, $\pi^{*}$ is optimal for the infinite-horizon discounted-cost case. 


\section{INFINITE-HORIZON AVERAGE COST}

In this section we study the infinite-horizon average-cost problem with i.i.d. demands. We first consider a finite-horizon model with no discounting $(\alpha=1)$ with a special terminal cost given by (15) at time $N$, and we assume independent and identical demands with distributions $F_{n}(x)=F(x)$.

THEOREM 4: Let

$$
S(w)=\beta w+F^{-1}\left(\frac{p}{p+h}\right) .
$$

The stationary base stock policy that orders up to $S(w)$ in state $(w, x)$ minimizes the $N$-period total cost.

Denote this stationary base stock policy as $\pi^{*}$. Let the expected cost per period of following policy $\pi$ for a finite-horizon $N$ starting from state $(w, x)$ be defined as

$$
g_{N}^{\pi}(w, x)=\frac{1}{N+1} E_{\pi}\left(\sum_{n=0}^{N-1} C_{n}\left(W_{n}, X_{n}, Y_{n}\right)+T\left(W_{N}, X_{N}\right) \mid W_{0}=w, X_{0}=x\right),
$$

and let $g_{N}(w, x)$ be the optimal expected cost per period for a finite-horizon $N$ starting in state $(w, x)$; that is,

$$
g_{N}(w, x)=\inf _{\pi} g_{N}^{\pi}(w, x)=\frac{V_{N}(w, x)}{N+1} .
$$

Theorem 4 implies that

$$
V_{N}(w, x)=V_{N}^{\pi^{*}}(w, x) .
$$

Hence, it follows that

$$
g_{N}(w, x)=g_{N}^{\pi^{*}}(w, x)
$$

We will show that

$$
\lim _{N \rightarrow \infty} \frac{1}{N+1}\left(E_{\pi^{*}}\left(T\left(W_{N}, X_{N}\right) \mid W_{0}=w, X_{0}=x\right)\right)=0
$$

and that

$$
\lim _{N \rightarrow \infty} \frac{1}{N}\left(E_{\pi^{*}}\left(\sum_{n=0}^{N-1} C_{n}\left(W_{n}, X_{n}, Y_{n}\right)\right) \mid W_{0}=w, X_{0}=x\right)
$$

exists. This will establish that $\pi^{*}$ minimizes the expected cost per period over the infinite horizon. To prove that the limit in (43) exists, we prove the following properties of $\left(W_{n}, X_{n}\right)$. 
THeOREM 5: Under the policy $\pi^{*},\left\{\left(W_{n}, X_{n}\right), n \geq 0\right\}$ is an irreducible, aperiodic, and positive recurrent Discrete Time Markov Chain (DTMC).

Proof: From the definition of the base stock policy and (1),

$$
\begin{aligned}
& X_{n+1}=\max \left(X_{n}, S\left(W_{n}\right)\right)-\beta W_{n}-\zeta_{n}, \\
& W_{n+1}=\delta_{n}\left(W_{n}+\min \left(\max \left(X_{n}, S\left(W_{n}\right)\right)-\beta W_{n}, \zeta_{n}\right)+\left[X_{n}\right]^{-}\right),
\end{aligned}
$$

where $S\left(W_{n}\right)$ is as in (37). This implies that $\left\{\left(W_{n}, X_{n}\right), n \geq 0\right\}$ is a DTMC. It is easy to show that it is irreducible and aperiodic. We prove the positive recurrence by using the Foster's criterion (Meyn and Tweedie [11]). We choose the following test function $v(W, X)=|X|+W$. Then

$$
\begin{aligned}
& E\left(v\left(W_{n+1}, X_{n+1}\right)-v\left(W_{n}, X_{n}\right) \mid W_{n}=w, X_{n}=x\right) \\
& \quad=E\left(W_{n+1}+\left|X_{n+1}\right| \mid W_{n}=w, X_{n}=x\right)-w-|x| .
\end{aligned}
$$

When $x \geq 0$, we have

$$
\begin{aligned}
& E\left(\left|X_{n+1}\right|-X_{n} \mid\left(W_{n}=w, X_{n}=x\right)\right) \\
& \quad=E\left(\left|\max \left(X_{n}, S\left(W_{n}\right)\right)-\beta W_{n}-\zeta_{n}\right|-X_{n} \mid W_{n}=w, X_{n}=x\right) \\
& \quad \leq \begin{cases}-\beta w+\mu & \text { if } x \geq S(w) \\
F^{-1}\left(\frac{p}{p+h}\right)+\mu-x & \text { otherwise }\end{cases} \\
& \leq F^{-1}\left(\frac{p}{p+h}\right)+\mu-x
\end{aligned}
$$

and

$$
\begin{aligned}
& E\left(W_{n+1}-W_{n} \mid\left(W_{n}=w, X_{n}=x\right)\right) \\
& =E\left(\delta\left(W_{n}+\min \left(\max \left(X_{n}, S\left(W_{n}\right)\right)-\beta W_{n}, \zeta_{n}\right)\right)-W_{n} \mid W_{n}=w, X_{n}=x\right) \\
& = \begin{cases}E\left(\delta(w+\min (x-\beta w, \zeta))-w \mid W_{n}=w, X_{n}=x\right) & \text { if } x \geq S(w) \\
E\left(\delta\left(w+\min \left(S(w)-\beta w, \zeta_{n}\right)\right)-w \mid W_{n}=w, X_{n}=x\right) & \text { otherwise }\end{cases} \\
& \leq \begin{cases}\delta \mu-(1-\delta) w & \text { if } x \geq S(w) \\
\delta \mu-(1-\delta) w & \text { otherwise }\end{cases} \\
& \leq-(1-\delta) w+\delta \mu .
\end{aligned}
$$


Therefore,

$$
\begin{aligned}
E(v( & \left.\left.W_{n+1}, X_{n+1}\right)-v\left(W_{n}, X_{n}\right) \mid W_{n}=w, X_{n}=x\right) \\
& =E\left(W_{n+1}+\left|X_{n+1}\right|-W_{n}-X_{n} \mid W_{n}=w, X_{n}=x\right) \\
& \leq F^{-1}\left(\frac{p}{p+h}\right)+(1+\delta) \mu-(1-\delta) w-x \\
& \leq F^{-1}\left(\frac{p}{p+h}\right)+(1+\delta) \mu-(1-\delta)(w+x) .
\end{aligned}
$$

The last expression is $<0$ if $(1-\delta)(w+x)>F^{-1}(p /(p+h))+(1+\delta) \mu$. When $x \leq 0$, we have

$$
\begin{aligned}
& E\left(\left|X_{n+1}\right|-\left|X_{n}\right| \mid W_{n}=w, X_{n}=x\right) \\
& \quad=E\left(\left|\max \left(X_{n}, S\left(W_{n}\right)\right)-\beta W_{n}-\zeta_{n}\right|+X_{n} \mid W_{n}=w, X_{n}=x\right) \\
& \quad \leq F^{-1}\left(\frac{p}{p+h}\right)+\mu+x \\
& \quad \leq 0 \quad \text { if } x \leq-\mu-F^{-1}\left(\frac{p}{p+h}\right)
\end{aligned}
$$

and

$$
\begin{aligned}
E\left(W_{n+1}-W_{n} \mid W_{n}=w, X_{n}=x\right) & \\
=E( & \delta\left(W_{n}+\min \left(\max \left(X_{n}, S\left(W_{n}\right)\right)-\beta W_{n}, \zeta_{n}\right)+\left[X_{n}\right]^{-}\right) \\
& \left.\quad-W_{n} \mid W_{n}=w, X_{n}=x\right) \\
=E( & \left.\delta\left(w+\min \left(F^{-1}\left(\frac{p}{p+h}\right), \zeta_{n}\right)-x\right)-w\right) \\
\leq \delta & (1-\delta) w-\delta x .
\end{aligned}
$$

Therefore,

$$
\begin{aligned}
& E\left(v\left(W_{n+1}, X_{n+1}\right)-v\left(W_{n}, X_{n}\right) \mid W_{n}=w, X_{n}=x\right) \\
& \quad=E\left(W_{n+1}+\left|X_{n+1}\right|-W_{n}+X_{n} \mid W_{n}=w, X_{n}=x\right) \\
& \quad \leq F^{-1}\left(\frac{p}{p+h}\right)+\mu+(1-\delta) x+\delta \mu-(1-\delta) w .
\end{aligned}
$$

The last expression is $<0$ if $(1-\delta)(w-x)>F^{-1}(p /(p+h))+(1+\delta) \mu$. 
Now define $A=\left\{(w, x):(1-\delta)(w+|x|) \leq F^{-1}(p /(p+h))+(1+\delta) \mu\right\}$. Note that $A$ is a finite set. Based on the above properties, we have shown that

$$
E\left(W_{n+1}+\left|X_{n+1}\right|-W_{n}-\left|X_{n}\right| \mid W_{n}=w, X_{n}=x\right)<0 \quad \text { if }(W, X) \notin A .
$$

Then Foster's criterion implies that $\left\{\left(W_{n}, X_{n}\right), n \geq 0\right\}$ is an irreducible and positive recurrent DTMC.

Next, we show the existence of the limit of $N$ period average total cost when $N$ goes to infinity.

Theorem 6: $\lim _{N \rightarrow \infty} E\left((1 / N) \sum_{n=0}^{N-1} C_{n}\left(W_{n}, X_{n}, Y_{n}\right) \mid W_{0}=w, X_{0}=x\right)$ exists and is finite for all $(w, x)$.

Proof: We obtain the total cost at period $n$ as

$$
\begin{aligned}
& E\left(C_{n}\left(W_{n}, X_{n}, Y_{n}\right) \mid W_{n}=w, X_{n}=x\right) \\
& \quad=E\left(c\left(y_{n}-X_{n}\right)+h\left(y_{n}-\beta W_{n}-\zeta_{n}\right)^{+}+p\left(y_{n}-\beta W_{n}-\zeta_{n}\right)^{-} \mid W_{n}=w, X_{n}=x\right) \\
& \quad \leq E\left(c\left(y_{n}-X_{n}\right)+h y_{n}+p\left(\zeta_{n}+\beta W_{n}\right) \mid W_{n}=w, X_{n}=x\right) \\
& \quad=E\left((c+h) \max \left(X_{n}, S\left(W_{n}\right)\right)+p\left(\zeta_{n}+\beta W_{n}\right)-c X_{n} \mid W_{n}=w, X_{n}=x\right) \\
& \quad=(c+h) E\left(\max \left(X_{n}, S\left(W_{n}\right)\right)\right)-c E\left(X_{n}\right)+p \mu+p \beta E\left(W_{n}\right) .
\end{aligned}
$$

When $x \geq 0$, we could rewrite the above quantity as

$$
\begin{aligned}
& E\left(C_{n}\left(W_{n}, X_{n}, Y_{n}\right) \mid W_{n}=w, X_{n}=x\right) \\
& \quad \leq(c+h) E\left(S\left(W_{n}\right)\right)-c E\left(X_{n}\right)+p \mu+p \beta E\left(W_{n}\right) \\
& \quad \leq(c+h)\left(\beta E\left(W_{n}\right)+F^{-1}\left(\frac{p}{p+h}\right)\right)+p \mu+p \beta E\left(W_{n}\right) \\
& \quad \leq(c+h) F^{-1}\left(\frac{p}{p+h}\right)+p \mu+(c+h+p) \beta\left(\frac{2 \mu}{1-\delta}+(w+x) \delta^{n}\right) .
\end{aligned}
$$

When $x \leq 0$, we get

$$
\begin{aligned}
& E\left(C_{n}\left(W_{n}, X_{n}, Y_{n}\right) \mid W_{n}=w, X_{n}=x\right) \\
& \quad \leq(c+h) E\left(X_{n}\right)-c E\left(X_{n}\right)+p \mu+p \beta E\left(W_{n}\right) \\
& \quad \leq p \mu+p \beta E\left(W_{n}\right) \\
& \quad \leq p \mu+p \beta\left(\frac{\mu}{1-\delta}+(w+x) \delta^{n}\right)
\end{aligned}
$$


Hence, we get

$$
\begin{aligned}
& E\left(C_{n}\left(W_{n}, X_{n}, Y_{n}\right) \mid W_{0}=w, X_{0}=x\right) \\
& \quad \leq(c+h) F^{-1}\left(\frac{p}{p+h}\right)+p \mu+(c+h+p) \beta\left(\frac{2 \mu}{1-\delta}+(w+x) \delta^{n}\right) \\
& \quad<\infty .
\end{aligned}
$$

The above bound along with the fact that $\left\{\left(W_{n}, X_{n}\right), n \geq 0\right\}$ is a positive recurrent and irreducible implies that

$$
\lim _{N \rightarrow \infty} E\left(\frac{1}{N} \sum_{n=0}^{N-1} C_{n}\left(W_{n}, X_{n}, Y_{n}\right) \mid W_{0}=w, X_{0}=x\right)
$$

exists and is finite.

Finally, we study the property of the special terminal cost and derive the optimal inventory policy to minimize the long-run average cost.

THEOREM 7: The stationary $w$-dependent base stock level policy $\pi^{*}$ minimizes the expected cost per period over the infinite horizon.

Proof: Using the terminal cost in (15) and using the argument in Theorem 3, we get

$$
\begin{aligned}
E_{\pi^{*}}( & \left.T\left(W_{N}, X_{N}\right) \mid W_{0}=w, X_{0}=x\right) \\
= & E_{\pi^{*}}\left(\frac{c \beta}{1-\delta} W_{N}-c X_{N} \mid W_{0}=w, X_{0}=x\right) \\
\leq & \left(\frac{2 \mu}{1-\delta}+(w+x) \delta^{N}\right) \frac{c \beta}{1-\delta}+c \mu .
\end{aligned}
$$

Hence,

$$
\lim _{N \rightarrow \infty} \frac{1}{N+1} E\left(T\left(W_{N}, X_{N}\right) \mid W_{0}=w, X_{0}=x\right)=0 .
$$

Since the $w$-dependent base stock policy minimizes the expected cost per period over $N$ periods for each $N$, it is clear that it minimizes the long-run average cost.

Note that the stationary $w$-dependent policy of Theorem 7 is independent of the parameters $c$ and $\delta$. This is so because all demands and their warranty-related subsequent demands will eventually be satisfied and there is no discounting. 


\section{EXTENSIONS OF THE PROPORTIONAL MODEL}

In Section 6 we derived optimal inventory replenishment policies assuming the following:

1. Proportional Warranty Expiration Model: a fixed fraction $1-\delta$ of the items under warranty a period $n$ go out of warranty by time $n+1$,

2. Proportional Failure Model: a fixed fraction $\beta$ of the items under warranty fail during that period.

In this section we relax some of these assumptions.

\subsection{Extensions of the Warranty Expiration Model}

Here we assume that a random fraction $\delta_{n}$ of items under warranty at time $n$ continue to be under warranty at time $n+1$ and that $\left\{\delta_{n}, n \geq 0\right\}$ is a sequence of i.i.d. random variables over $(0,1)$ with common c.d.f. $G(\cdot)$ and mean $\delta$. We keep the assumption of constant $\beta$ for all periods $n$. Then the number of items under warranty change according to

$$
\begin{aligned}
& W_{n+1}=\delta_{n}\left[(1-\beta) W_{n}+\min \left(Y_{n}, \beta W_{n}+\zeta_{n}\right)+\left[X_{n}\right]^{-}\right], \\
& X_{n+1}=Y_{n}-\zeta_{n}-\beta W_{n} .
\end{aligned}
$$

With this modification, most of the analysis of the previous sections remains valid. We mention only those parts that change. Equation (13) for $G_{n, N}(w, x, y)$ becomes

$$
\begin{aligned}
& G_{n, N}(w, x, y) \\
& =c y-c x+\alpha L_{n}(w, y) \\
& \quad+\alpha \int_{0}^{1}\left[\int_{0}^{y-\beta w} V_{n+1, N}\left(u\left(w+\zeta+[x]^{-}\right), y-\beta w-\zeta\right) f_{n}(\zeta) d \zeta\right. \\
& \left.\quad+\alpha \int_{y-\beta w}^{\infty} V_{n+1, N}\left(u\left((1-\beta) w+y+[x]^{-}\right), y-\beta w-\zeta\right) f_{n}(\zeta) d \zeta\right] d G(u) .
\end{aligned}
$$

Theorem 1 remains valid with the corresponding modifications. Hence, we see that the $w$-dependent base stock policy that orders up to $S_{n}(w)$, as defined in (17), in period $n$ in state $(w, x)$ remains optimal. We need to just interpret $\delta$ of (17) as the expected value of $\delta_{n}$.

Note that the intuitive model in which each model goes out of warranty with a given probability in each period is not a special case of random $\delta$ model. Thus, the 
above policy will not be valid in this case. We will study more realistic warranty expiration models in a separate article in the near future.

\subsection{Extensions of the Failure Model}

Next, assume that $\delta_{n}$ are i.i.d. random variables with support on $[0,1]$ and common mean $\delta$. Furthermore, assume that a random fraction $\beta_{n}$ of items under warranty at time $n$ fail during that period. Assume that $\left\{\beta_{n}, n \geq 0\right\}$ are i.i.d. random variables with support on $[0,1]$ and common mean $\beta$. Unfortunately, the results about optimal policies derived earlier fail to hold in this case. Thus, there might not be a $w$-dependent optimal policy in this case. Hence, we develop a heuristic to handle this case.

Now suppose $W_{n}=w$. Then the total demand in period $n$ is given by $D_{n}=$ $\zeta_{n}+\beta_{n} w$. Let $F_{n, w}$ be the distribution of $D_{n}$ and define

$$
S_{n}(w)=F_{n, w}^{-1}\left(\frac{\bar{p}-c(1-\alpha)}{\bar{p}+h}\right),
$$

where $\bar{p}$ is as defined in (16). Now consider a policy that orders up to $S_{n}(w)$ when the state is $(w, x)$ in period $n$. Clearly, this policy reduces to the provable optimal policy when $\beta_{n}=\beta$, a fixed constant for all $n$. One can also show that this policy is optimal for a one-period problem $(N=1)$. Hence, we propose this policy as a heuristic policy to be used when our constant $\beta$ model fails to hold.

One advantage of this model is that it is fairly straightforward to estimate $\delta$ and $\beta$ from observable data.

\section{MODEL WITH EMERGENCY SUPPLY}

In this section we study the emergency supply model where the demands cannot be backlogged. Instead, the manufacturer has to satisfy the unmet demands by purchasing the items from an emergency supplier. We use $p$ as the per-unit emergency purchase cost where $p>c$. Note that there are no shortages in this model. As earlier, let $\beta$ be the constant failure failure fraction and $1-\delta$ be the constant warranty expiration rate in period $n$. The dynamic of the system is given by

$$
\begin{aligned}
& X_{n+1}=\max \left(Y_{n}-\beta W_{n}-\zeta_{n}, 0\right), \\
& W_{n+1}=\delta\left(W_{n}+\zeta_{n}\right) .
\end{aligned}
$$

In this section we study the finite-horizon ETDC problem with $N$ periods and terminal cost $T(w, x)$ and show how to compute $V_{N}(w, x)$ of (5). First, define $V_{n, N}(w, x)$ to be the optimal ETDC over periods $n, n+1, \ldots, N$ starting with $W_{n}=w$ and $X_{n}=x$. Let $G_{n, N}(w, y)-c x$ be the ETDC over periods $n, n+1, \ldots, N$ given $W_{n}=w$ and $Y_{n}=y$. Then the standard dynamic programming recursion yields 


$$
\begin{aligned}
V_{N, N}(w, x)= & T(w, x) \\
G_{n, N}(w, y)= & c y+\alpha L_{n}(w, y)+\alpha \int_{0}^{y-\beta w} V_{n+1, N}(\delta(w+\zeta), y-\beta w-\zeta) f_{n}(\zeta) d \zeta \\
& +\alpha \int_{y-\beta w}^{\infty} V_{n+1, N}(\delta(w+\zeta), 0) f_{n}(\zeta) d \zeta, \quad 0 \leq n \leq N-1, \\
V_{n, N}(w, x)= & \min _{y \geq x}\left\{G_{n, N}(w, y)\right\}-c x, \quad n=0,1, \ldots, N-1,
\end{aligned}
$$

where $L_{n}(w, y)$ is as in (2). Then we have

$$
V_{N}(w, x)=V_{0, N}(w, x)
$$

We will use the following special terminal cost function:

$$
T(w, x)=-c x+\frac{c \beta w}{1-\alpha \delta} .
$$

The above terminal cost is easily explained if we assume that any leftover inventory at period $N$ can be returned at the original purchase price $c$. Furthermore, all of the warranty claims arising out of the $w$ items under warranty have to be covered. With this terminal operation, the first term in (50) reflects the cost of processing the terminal inventory $x$ and the second term is the cost of the warranty claims arising from the $w$ items left under warranty at the end of the horizon. Note that this terminal cost is the same as the one in (15), except that there is no $[x]^{-}$term now since there are no backlogs. We need the following notations. For $n=0,1, \ldots, N-1$, define

$$
\begin{aligned}
S_{n}(w) & =\beta w+F_{n}^{-1}\left(\frac{p-c}{p+h-c}\right), \\
\bar{L}_{n}(w, y) & =(h-c) \int_{0}^{y-\beta w}(y-\beta w-\zeta) f_{n}(\zeta) d \zeta+p \int_{y-\beta w}^{\infty}(\zeta-y+\beta w) f_{n}(\zeta) d \zeta, \\
\tau_{n}(w, y) & =\frac{y-\beta w-\beta \delta w-F_{n}^{-1}\left(\frac{p-c}{p+h-c}\right)}{1+\delta \beta}, \\
\Delta_{n} & =\alpha\left(\frac{c \beta}{1-\alpha \delta} \delta\right) \mu_{n}+c F_{n}^{-1}\left(\frac{p-c}{p+h-c}\right)+\alpha \bar{L}_{n}\left(w, S_{n}(w)\right), \\
\mu_{n} & =E\left[\zeta_{n}\right]=\int_{0}^{\infty}\left(1-F_{n}(\zeta)\right) d \zeta .
\end{aligned}
$$


Also, define

$$
\begin{aligned}
& H_{N}(w, x)=0, \\
& H_{n}(w, x)=\left\{\begin{array}{ll}
0 & \text { if } x \leq S_{n}(w) \\
G_{n, N}(w, x)-G_{n, N}\left(w, S_{n}(w)\right) & \text { otherwise }
\end{array} \text { for } 0 \leq n \leq N-1 .\right.
\end{aligned}
$$

Note that $\Delta_{n}$ of (52) is independent of $w$, as earlier. This fact is important in the following analysis.

THEOREM 8: Suppose the demands in each period are stochastically increasing; that is,

$$
F_{n}(x) \geq F_{n+1}(x) \text { for } n=0,1, \ldots, N-2 \text {. }
$$

Then

(i)

$$
\begin{aligned}
G_{n, N}(w, y)= & c y+\alpha \bar{L}_{n}(w, y)+\sum_{i=n+1}^{N-1} \alpha^{i-n} \Delta_{i}+\alpha\left(\frac{c \beta}{1-\alpha \delta} \delta\right) w \\
& +\alpha\left(\frac{c \beta}{1-\alpha \delta} \delta\right) \mu_{n} \\
& +\alpha \int_{0}^{\tau_{n+1}(w, y)} H_{n+1}(\delta(w+\zeta), y-\beta w-\zeta) f_{n}(\zeta) d \zeta
\end{aligned}
$$

for $n=0, \ldots, N-1$. Furthermore, $y=S_{n}(w)$ minimizes $G_{n}(w, y)$ for $n=$ $0, \ldots, N-1$ and $G_{n, N}(w, y)$ increases with respect to $y$ when $y \geq S_{n}(w)$.

(ii)

$$
\int_{0}^{\tau_{n}(w, y)} H_{n}(\delta(w+\zeta), y-\beta w-\zeta) f_{n-1}(\zeta) d \zeta
$$

is an increasing function of $y$ when $y \geq S_{n-1}(w)$ for a given $w$ for $n=1, \ldots, N-1$.

(iii)

$$
V_{n, N}(w, x)=T(w, x)+\sum_{i=n}^{N-1} \alpha^{i-n} \Delta_{i}+H_{n}(w, x)
$$

for $n=0, \ldots, N-1$.

The proof follows from a series of claims using backward induction. 
Claim 1: Parts (i), (ii), and (iii) hold for $N-1$.

PROOF:

$$
\begin{aligned}
G_{N-1, N}(w, y)= & c y+\alpha L_{N-1}(w, y) \\
& +\alpha \int_{0}^{y-\beta w}\left[\frac{c \beta}{1-\alpha \delta} \delta(w+\zeta)-c(y-\beta w-\zeta)\right] f_{N-1}(\zeta) d \zeta \\
& +\alpha \int_{y-\beta w}^{\infty}\left[\frac{c \beta}{1-\alpha \delta} \delta(w+y-\beta w)\right] f_{N-1}(\zeta) d \zeta \\
= & c y+\alpha\left(\frac{c \beta}{1-\alpha \delta} \delta\right)\left(w+\mu_{N-1}\right)+\alpha \bar{L}_{N-1}(w, y) .
\end{aligned}
$$

It is easy to see that $y=S_{N-1}(w)=\beta w+F_{N-1}^{-1}((p-c) /(p+h-c))$ minimizes $G_{N-1, N}(w, y)$ for all $x$. Hence, $G_{N-1, N}(w, y)$ increases with respect to $y$ when $y \geq$ $S_{N-1}(w)$. The rest of the proof follows along the same lines as the proof of Claim 1 in the proof of Theorem 1 .

Claim 2: If parts (i), (ii), and (iii) of Theorem 1 hold for $n \leq N-1$, then they hold for $n-1$.

Proof: The proof follows along the same lines as the proof of Claim 2 in the proof of Theorem 1 .

From Theorem 8 it follows that the optimal policy in period $n$ in state $(w, x)$ is to order up to $S_{n}(w)$ as given in (51). This is called a $w$-dependent base stock inventory replenishment policy. Note that this policy is independent of $\alpha$ and $\delta$. This is a consequence of the emergency supply assumption.

When the demands $\left\{\zeta_{n}, n \geq 0\right\}$ are i.i.d. with common c.d.f. $F(\cdot)$, we get an optimal stationary policy that orders up to

$$
S(w)=\beta w+F^{-1}\left(\frac{p-c}{p+h-c}\right)
$$

in state $(w, x)$ in any period. One can show that this policy also minimizes the infinitehorizon ETDC and also the infinite-horizon cost per period, following the same arguments in Sections 5 and 6. We can also extend the failure and the warranty expiration models along the same lines as in Section 7.

\section{COMPUTATIONAL RESULTS}

In this section we numerically investigate the benefits of using an integrated inventory policy over the current ad hoc policy used by the firm where they take only new demand into consideration; that is, they order up to 


$$
S=F^{-1}\left(\frac{p-(1-\alpha) c}{p+h}\right) .
$$

Although the optimal policy is known, computing its performance analytically is intractable. Hence, we use simulation to evaluate the performance of these two policies.

We compare the performance over 343 problem instances with the following parameter values: $c=2, \delta=0.96, \alpha=0.95$. Then we vary $h=0.05,0.1,0.15,0.2$, $0.25,0.30, p=8,10,12,15,20,25,30$, and $\beta=0.01,0.05,0.1,0.15,0.2,0.25,0.3$. In these instances, we compute the total $N$ periods $(N=100)$ discounted cost of the two policies and average it across 1000 simulations. In all of our experiments, we consider a stationary and uniform demand distribution over 0 to 100 items in every period. In the following passages we highlight our key insights.

Across all of these experiments, the average-cost improvement due to the integrated policy was $53.26 \%$ with a maximum improvement of $86.64 \%$ (see Tables $1-3)$. For example, from Table 1 we see that when $p=8$, the average improvement in the cost over all the cases with different values of $h$ and $\beta$ (given above) is

TABLE 1. Average Cost Improvement over $p$

\begin{tabular}{lrrcrcrr}
\hline & $p=8$ & 10 & 12 & 15 & 20 & 25 & 30 \\
\hline Avg. \% & 40.94 & 45.35 & 48.87 & 53.07 & 58.14 & 61.82 & 64.61 \\
Max. \% & 62.45 & 67.65 & 71.7 & 76.08 & 81.07 & 84.3 & 86.64 \\
Min. \% & 1.11 & 1.33 & 1.65 & 2.09 & 2.65 & 3.28 & 4.02 \\
\hline
\end{tabular}

TABLE 2. Average Cost Improvement over $h$

\begin{tabular}{lccccccr}
\hline & $h=0.01$ & 0.05 & 0.1 & 0.15 & 0.2 & 0.25 & 0.3 \\
\hline Avg. \% & 53.63 & 53.50 & 53.38 & 53.26 & 53.12 & 53.01 & 52.88 \\
Max. \% & 86.64 & 86.52 & 86.47 & 86.34 & 86.33 & 86.3 & 86.26 \\
Min. \% & 1.23 & 1.2 & 1.13 & 1.12 & 1.11 & 1.11 & 1.11 \\
\hline
\end{tabular}

TABLE 3. Average Cost Improvement over $\beta$

\begin{tabular}{llllllll}
\hline & $\beta=0.01$ & 0.05 & 0.1 & 0.15 & 0.2 & 0.25 & 0.3 \\
\hline Avg. \% & 2.39 & 29.99 & 54.47 & 65.71 & 70.98 & 73.80 & 75.46 \\
Max. \% & 4.29 & 45.1 & 70.48 & 79.66 & 83.54 & 85.46 & 86.64 \\
Min. \% & 1.11 & 17.2 & 37.93 & 50.05 & 56.18 & 59.82 & 61.9 \\
\hline
\end{tabular}




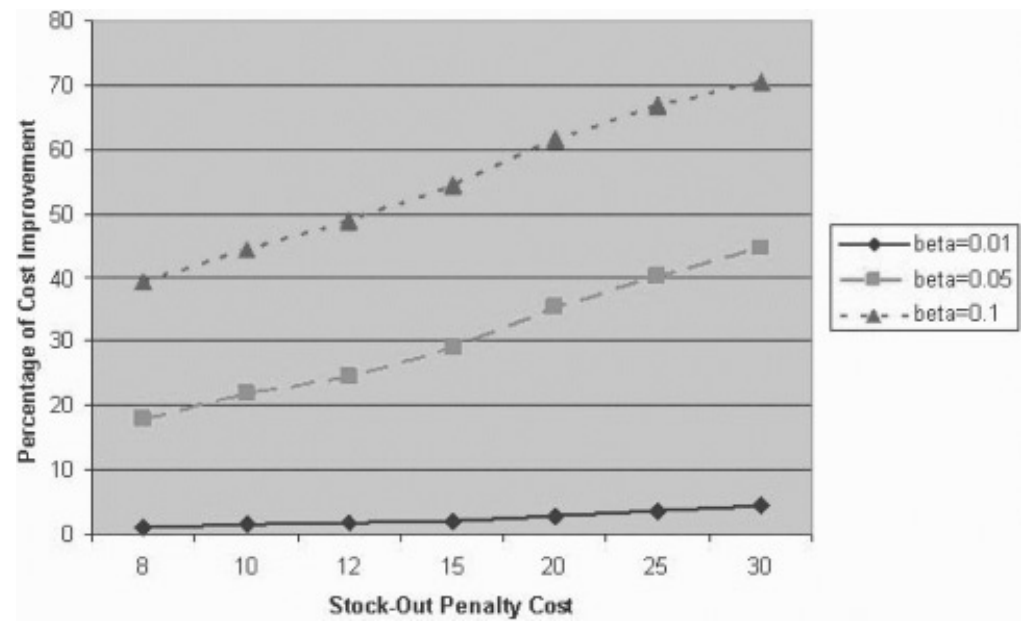

Figure 1. The cost improvement with increasing stock-out penalty cost rate $p$.

12.7\%. From Figures 1 and $2(h=0.01)$ we observe the cost improvement monotonically increases with the stock-out penalty $\operatorname{cost} p$ and $\beta$ when all other parameters are fixed. We have not plotted the improvement as a function of $h$, since the holding costs are not significant components of the total costs in either policy.

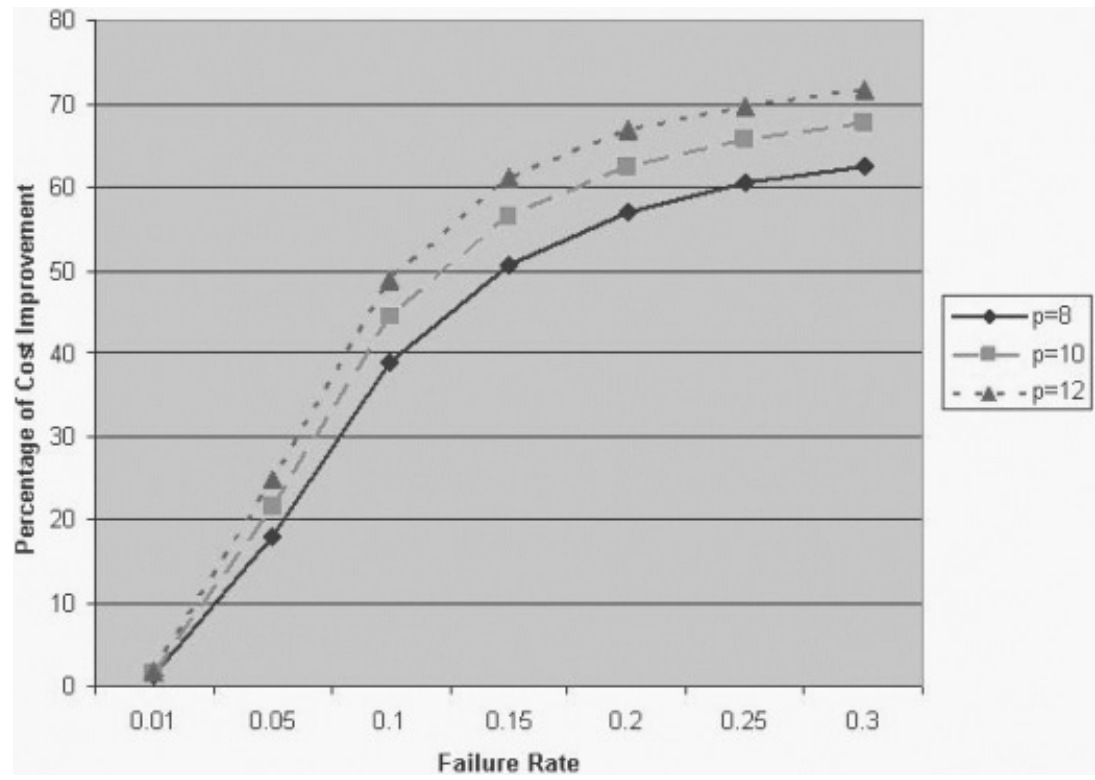

Figure 2. The cost improvement with increasing failure rate $\beta$. 


\section{CONCLUSION}

Motivated by the inventory planning issues faced by a seller of items under warranty, in this article we analyzed a setting in which the seller faces demand from two sources: new demand and demand to replace failed items under warranty. We considered backlogging and emergency supply cases and studied both the discounted-cost and the average-cost cases. We proved the optimality of the $w$-dependent base stock ordering policy where the base stock level is a function of $w$, the number of items currently under warranty. For the special case where an i.i.d. fraction of sold items go out of warranty every period and the demand for new products is stationary, we prove the optimality of a stationary $w$-dependent base stock policy for finite- and infinitehorizon cases. Through our computational study, we provide interesting insights on the benefits of using an integrated policy and show that, on average, this leads to a $53 \%$ improvement over an inventory policy that only considers new demands.

\section{Acknowledgment}

This research was partially supported by NSF grant DMI-0223117.

\section{References}

1. Baker, R.C. \& Urban, T.L. (1988). A deterministic inventory system with an inventory-leveldependent demand rate. Journal of the Operational Research Society 39(9): 823-831.

2. Blischke, W.R. \& Murthy, D.N.P. (1994). Warranty cost analysis. New York: Marcel Dekker.

3. Cohen, M.A., Nahmias, S., \& Pierskalla, W.P. (1980). A dynamic inventory system with recycling. Naval Research Logistics Quarterly 27(2): 289-296.

4. DeCroix, G.A. (2006). Optimal policy for a multi-echelon inventory system with remanufacturing. Operations Research 54: 532-544.

5. Djamaludin, I., Murthy, D.N.P., \& Wilson, R.J. (1994). Quality control through lot sizing for items sold with warranty. International Journal of Production Economics 33: 97-107.

6. Feinberg, E.A. \& Lewis, M.E. (2005). Optimality of four-threshold policies in inventory systems with customer returns and borrowing/storage options. Probability in Engineering and Informational Sciences 19: 45-71.

7. Guide, D. \& Wassenhove, L.N.V. (2003). Business aspects of closed loop supply chains. Pittsburgh: Carnegie Mellon University Press.

8. Inderfurth, K. (1997). Simple optimal replenishment and disposal policies for a product recovery system with leadtimes. Operative Research Spektrum 19: 111-122.

9. Kelle, P. \& Silver, E.A. (1989). Forecasting the returns of reusable containers. Journal of Operations Management 8(1): 17-35.

10. Khmelnitsky, E. \& Gerchak, Y. (2001). Optimal control approach to production systems with inventory-level-dependent demand. IEEE Transactions on Automatic Control 47 (2): 289-292.

11. Meyn, S.P. \& Tweedie, R.L. (1993). Markov chains and stochastic stability. London: Springer-Verlag.

12. Porteus, E.L. (1986). Optimal lot sizing, process quality improvement and setup cost reduction. Operations Research 34(1): 137-144.

13. Simpson, V.P. (1978). Optimum solution structure for a repairable inventory problem. Operations Research 26(2): 270-281.

14. Swaminathan, J.M. \& Tayur, S.R. (2003). Tactical planning models for supply chain management. In A.G. de Kok \& S.C. Graves (eds.), Handbooks in ORMS, 11: Supply chain management: Design, coordination and operation. New York: Elsevier. pp. 423-456.

15. Wang, Ch.H. \& Sheu, Sh.H. (2003). Optimal lot sizing for products sold under free-repair warranty. European Journal of Operational Research 149(1): 131-141.

16. Yuan, X.M. \& Cheung, K.L. (1998). Modeling returns of merchandise in an inventory system. Operations Research Spektrum 20(3): 147-154. 